\title{
Erratum to: Energy Conservation via Antenna Scheduling in Fiber-Connected Femto Base Stations
}

\author{
Haoming Li • Alireza Attar • Victor C. M. Leung
}

Published online: 8 November 2013

(C) Springer Science+Business Media New York 2013

\section{Erratum to: Mobile Netw Appl (2012) 17:685-694 DOI 10.1007/s11036-012-0395-x}

In the original version of this article, the channel bandwidth $B$ was missing in the definition of energy efficiency and the total number of users was inadvertently removed in the simulation scripts of energy efficiency. As a result, the following corrections are needed.

The sentence in the beginning of Section 3, "Define energy efficiency as $\eta_{e}=\eta_{s} / P_{\text {avg }}$, where $\eta_{s}$ is", should be written as "Define energy efficiency as $\eta_{e}=B \cdot \eta_{s} / P_{\text {avg }}$, where $B$ is the channel bandwidth, $\eta_{s}$ is". Equations (10) and (18) should have a multiplication constant $B$ in their numerators:

$\eta_{e}=B\left(c_{1} \ln P_{A E}+c_{2}\right) /\left(B P_{A E} / \gamma+c_{3}\right)$,

$\left.\frac{\partial^{2} \eta_{e}}{\partial P_{A E}^{2}}\right|_{P_{A E}=P^{*}}=\frac{-B \gamma c_{1}}{\left(P^{*}\right)^{2}\left(B P^{*}+c_{3} \gamma\right)}<0$

In the right subfigure of Figure 3, the unit of Y-axis should be "Mbits/Joule", i.e., $10^{6}$ bits/Joule, and the Y-axis labels should be " $24,48,72,96,120,144,168,192,216,240,264$ ". The correct subfigure is shown as follows:

The online version of the original article can be found at http:// dx.doi.org/10.1007/s11036-012-0395-x.

\section{H. Li $(\bowtie) \cdot$ A. Attar $\cdot$ V. C. M. Leung}

Department of Electrical and Computer Engineering, The University of British Columbia, 5500-2332 Main Mall, Vancouver,

BC V6T1Z4, Canada

e-mail: hlih@ece.ubc.ca
A. Attar
e-mail: attar@ece.ubc.ca
V. C. M. Leung
e-mail: vleung@ece.ubc.ca

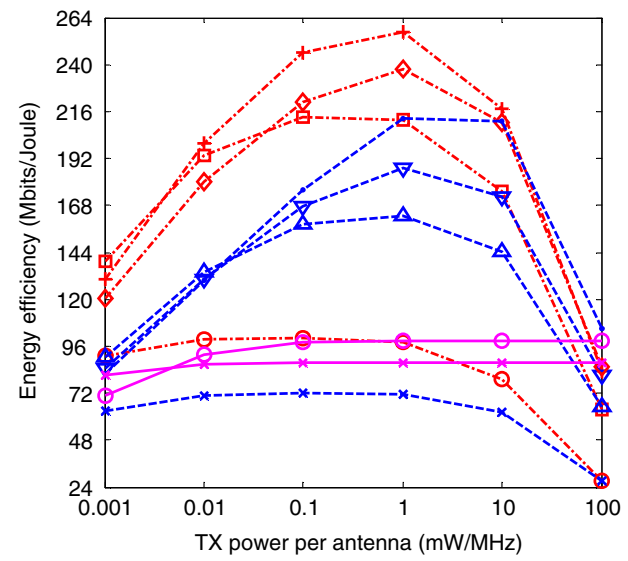

In Fig. 4, the unit of Y-axis should be "Mbits/Joule" and the Y-axis labels should be " $0,48,96,144,192,240,288$ ". The correct Fig. 4 is shown as follows:

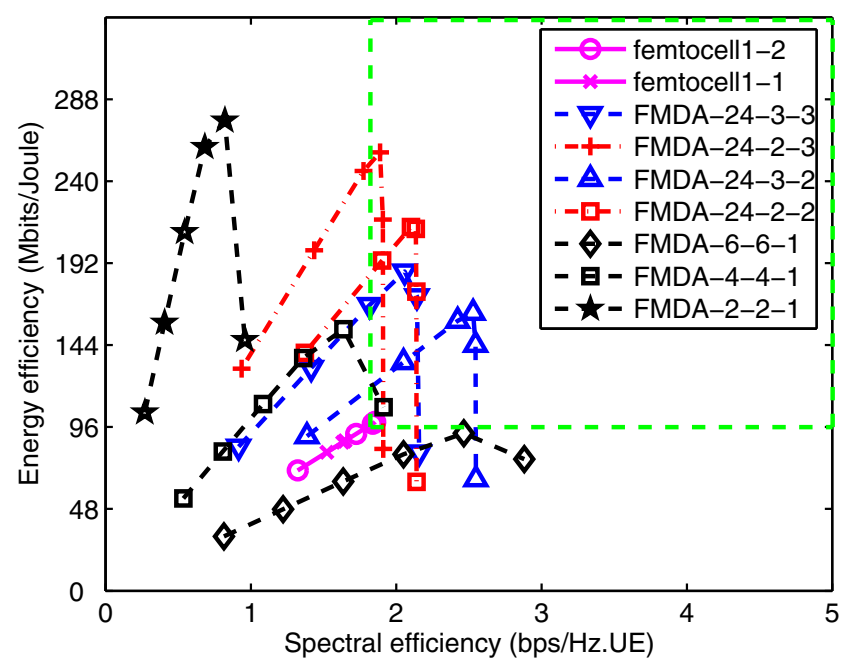

Fig. 4 Energy efficiency vs. spectral efficiency under different $P_{A E}\left(10^{-3} \sim 10^{2} \mathrm{~mW} / \mathrm{MHz}\right)$ 
As the channel bandwidth in this article is fixed at $10 \mathrm{MHz}$ and the total number of users is fixed at 48 , the above errors do not affect the optimization results in Section 3 or the energy efficiency comparisons in Section 5. We apologize to our readers for these errors. 